\title{
Relationship between Conjunctival Intraepithelial Dendritic Melanocytes and Nevocytes
}

\author{
Frederick A. Jakobiec ${ }^{a} \quad$ Paula Cortes Barrantes ${ }^{a} \quad$ Mary E. Aronow ${ }^{b}$ \\ ${ }^{a}$ David G. Cogan Laboratory of Ophthalmic Pathology, Massachusetts Eye and Ear/Harvard Medical School, \\ Boston, MA, USA; ${ }^{b}$ Retina Service, Department of Ophthalmology, Massachusetts Eye and Ear/Harvard Medical \\ School, Boston, MA, USA
}

\section{Established Facts}

- Dendritic melanocytes and nevocytes are morphologically distinguishable cell types.

- Dendritic melanocytes transfer pigment to surrounding keratinocytes.

- Nevocytes lack dendrites and synthesize malformed melanosomes that are not transferred.

- Nevocytes (responsible for common acquired nevi/moles) are regarded as a cell type different from dendritic melanocytes. From their initial origins in the neural crest, these two cell types are believed to migrate separately to their respective destinations.

\section{Novel Insights}

- An intraepithelial dendritic melanocytic proliferation (also referred to as conjunctival intraepithelial neoplasia and previously as primary acquired melanosis) was discovered to have an early compound nevus characterized by nests of nevocytes both within the epithelium and in the substantia propria.

- Certain factors (e.g., ultraviolet light exposure) may cause dendritic melanocytes to transform into rounded nevocytes that generate common acquired nevocytic nevi.

- Conjunctival junctional and compound nevi in adults can sometimes arise from scattered intraepithelial conjunctival dendritic melanocytes, as in the current lesion.

\section{Keywords}

Conjunctiva $\cdot$ Primary acquired melanosis · Intraepithelial melanocytic neoplasia . Dendritic melanocyte .

Nevus cell $\cdot$ Nevomelanocyte $\cdot$ Origin of nevocyte $\cdot$ Immunohistochemistry $\cdot$ MART-1 $\cdot \mathrm{HMB}-45 \cdot \mathrm{Ki}-67 \cdot \mathrm{SOX}-10$

\begin{abstract}
A 52-year-old male presented with a perilimbal-epibulbar, flat, pigmented lesion of 7 months' duration. Microscopic evaluation disclosed a proliferation of intraepithelial dendritic melanocytes without frank atypia, a lesion formerly termed "primary acquired melanosis." Within the lesion there were also intraepithelial basal junctional nevocytic
\end{abstract}

karger@karger.com

(C) 2020 S. Karger AG, Basel

www.karger.com/oop

Karger $\stackrel{2}{\circ}$
Frederick A. Jakobiec, MD, DSc

David G. Cogan Laboratory of Ophthalmic Pathology, Department of Ophthalmology Massachusetts Eye and Ear Infirmary, 243 Charles Street, Suite 328 Boston, MA 02114 (USA)

Fred_Jakobiec@meei.harvard.edu 
nests and occasional small subepithelial nevocytic clusters which were positive for MART-1, HMB-45, and SOX-10 and negative for Ki-67. This remarkable lesion was suggestive of dendritic melanocytes transforming into rounded nevocytes lacking dendrites. The embryologic and biologic implications of these findings are explored, notably in regard to the unusual acquisition in mature adults of common nevomelanocytic nevi.

(c) 2020 S. Karger AG, Basel

\section{Introduction}

Intraepithelial dendritic melanocytes and melanocytic nevus cells (nevocytes) of the skin and mucosae, including the conjunctiva, have been considered by many experts to be totally separate cell lines [1-6], although differences of opinion do exist. We report an unusual con- junctival pigmented lesion in an adult, which presented as an intraepithelial melanocytic proliferation with a secondary common acquired nevus evolving within it. This observation challenges the tenet that dendritic melanocytes and nevocytes are two totally unrelated melanocytic cell lines. The present report explores the embryologic and biologic implications of this phenomenon.

\section{Case Report}

A 52-year-old Caucasian male presented for a second opinion regarding a conjunctival lesion of his left eye. The patient had been aware of a discoloration at the temporal limbus for approximately 7 months. He had no significant past medical history and there was no personal or family history of melanoma. Due to growth and darkening of the lesion, he sought evaluation by an ophthalmologist at an outside hospital. Because there were no unusual features observed, a clinical pre-biopsy photograph of the lesion was not taken. Visual acuity with spectacle correction measured 20/20 in

Fig. 1. Histopathologic and immunohistochemical features of a conjunctival pigmented lesion (intraepithelial melanocytic proliferation). a Top panel: routine staining of the excised specimen shows an absence of tumor nodules, only a flat mucosal membrane. Bottom panel: MART-1 staining reveals a population of positive staining of cells in the basal layer of the epithelium, as well as two rounded intraepithelial cellular nests (arrows). b Increased numbers of pigmented cells are located in the basal layer of the epithelium. Note the prominent elastotic material in the substantia propria (arrow). c More pronounced basal melanocytic proliferation. These cells have not percolated to higher levels of the epithelium. d A cluster of basal melanocytes has retracted from the surrounding keratinocytes (arrows) to occupy a small cleft (pseudojunctional nest). e MART-1 reveals a dense population of basal melanocytes that make side-by-side contact. f There is a focal pileup of mostly basal and suprabasal melanocytes displaying tangles of their dendritic processes (arrows). a Hematoxylin and eosin (HE). Top and bottom panels, $\times 2$. b HE. $\times 40$. c HE. $\times 40$. d HE. $\times 40$. e, f Immunoperoxidase, red chromogen, hematoxylin counterstain. e $\times 60$. f Immunoperoxidase. $\times 40$.
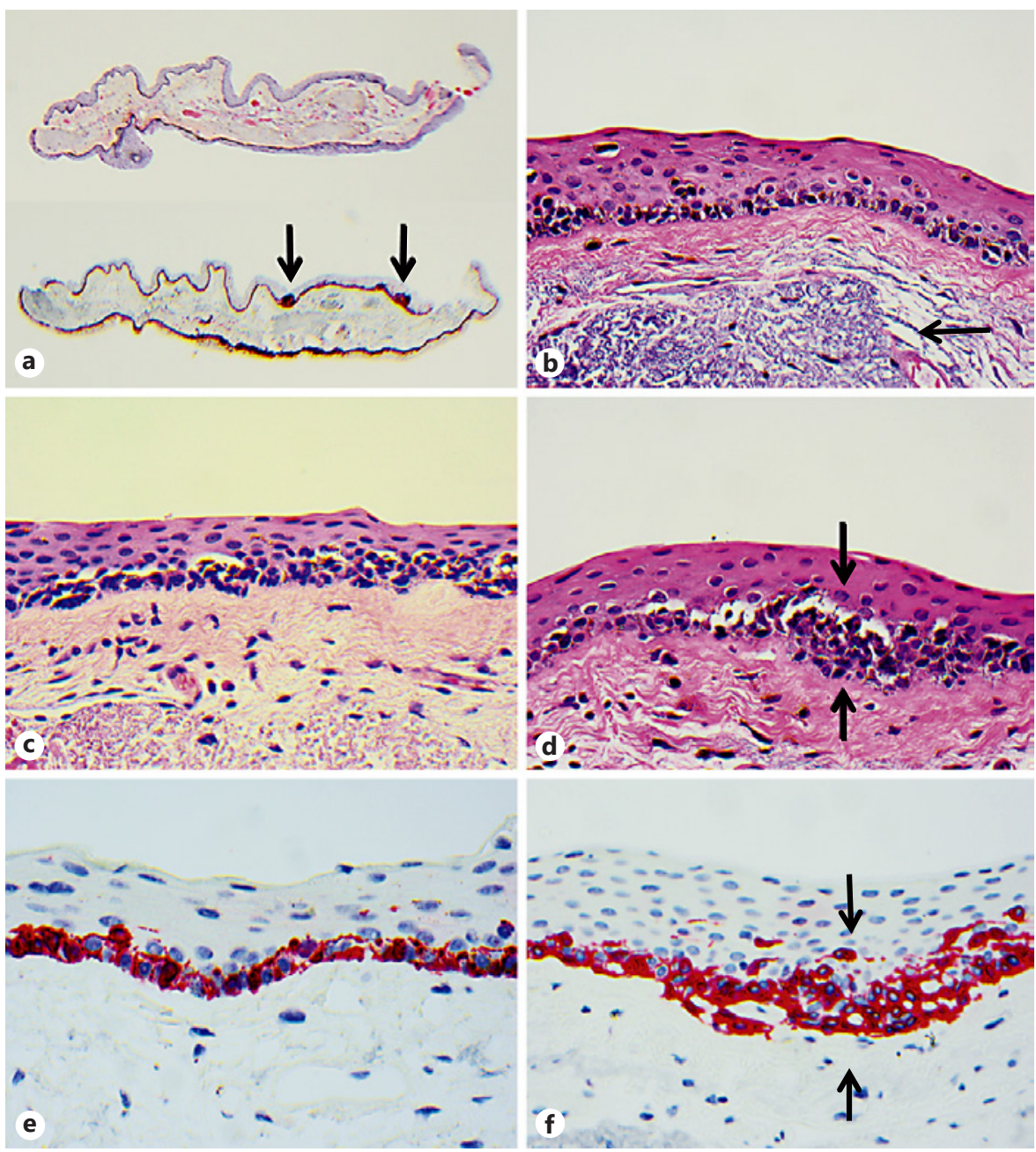
both eyes. The intraocular pressures were $15 \mathrm{~mm} \mathrm{Hg}$ in the right eye and $18 \mathrm{~mm} \mathrm{Hg}$ in the left. Anterior segment examination was normal for the right eye. Examination of the left eye revealed a small mobile pigmented perilimbal conjunctival lesion measuring $0.5 \times 0.3 \times 0.2 \mathrm{~cm}$. Dilated fundus examination was normal in both eyes. Given the patient's age at the time of first detection of the lesion and its alleged increase in size, an excisional biopsy was recommended. During 5 months of follow-up, the lesion has not recurred; careful follow-up examinations have been scheduled for an additional year.

\section{Results}

\section{Histopathologic and Immunochemical Findings}

Excised at an outside hospital, the specimen was described as a tan-brown fragment of tissue measuring $0.4 \times 0.2 \times 0.1 \mathrm{~cm}$ submitted in toto (Fig. 1a, top and bot- tom panels). On low-power microscopy, within the epithelium there were varying densities of basally situated pigmented dendritic melanocytes (Fig. 1b). These cells ranged in disposition from a back-to-back arrangement (Fig. 1c) to a noncontiguous diaspora of cells near the lateral surgical edges. There were also small, loose clusters (Fig. 1d) of intraepithelial melanocytes in rounded clearcut nevocytic groups (theques) within small cavities. These cells lacked dendrites and were mutually adhesive.

Immunochemical staining with MART-1 defined the dendritic shape of the basal melanocytes and separated them from the basal (germinal) keratinocytes (Fig. 1e). Focal areas of pronounced melanocytic dendritic cell proliferations were identified basally and suprabasally (Fig. 1f). On occasion, the dendritic melanocytes drifted to the mid-level of the epithelium with visible elongated dendrites (Fig. 2a).
Fig. 2. Immunostaining results for intraepithelial melanocytes. a Dense proliferation of basal melanocytes and their dendrites demonstrated with MART-1. Several dendritic melanocytes are observed in the midepithelium. b A MART-1-positive, tightly organized basal cellular cluster has arisen in a segment of intraepithelial melanocytic proliferation. c Another junctional nest is composed of compact melanocytes without discernible dendrites, as demonstrated by their smooth outlines. Dendritic melanocytes are found on both sides of the nest. d HMB-45 immunostains both the basal dendritic melanocytes and the nevocytes in the junctional nests. e A nevocytic nest has dropped off into the superficial substantia propria while maintaining a focal, faint contact with an overlying intraepithelial nevoid nest. f Intraepithelial basal dendritic melanocytes display SOX-10 nuclear positivity. Immunoperoxidase red chromogen, hematoxylin counterstain. a-c $\times 40, \times 20, \times 40$. e, $\mathbf{f} \times 40, \times 40$. d Diaminobenzidine chromogen. $\times 60$.

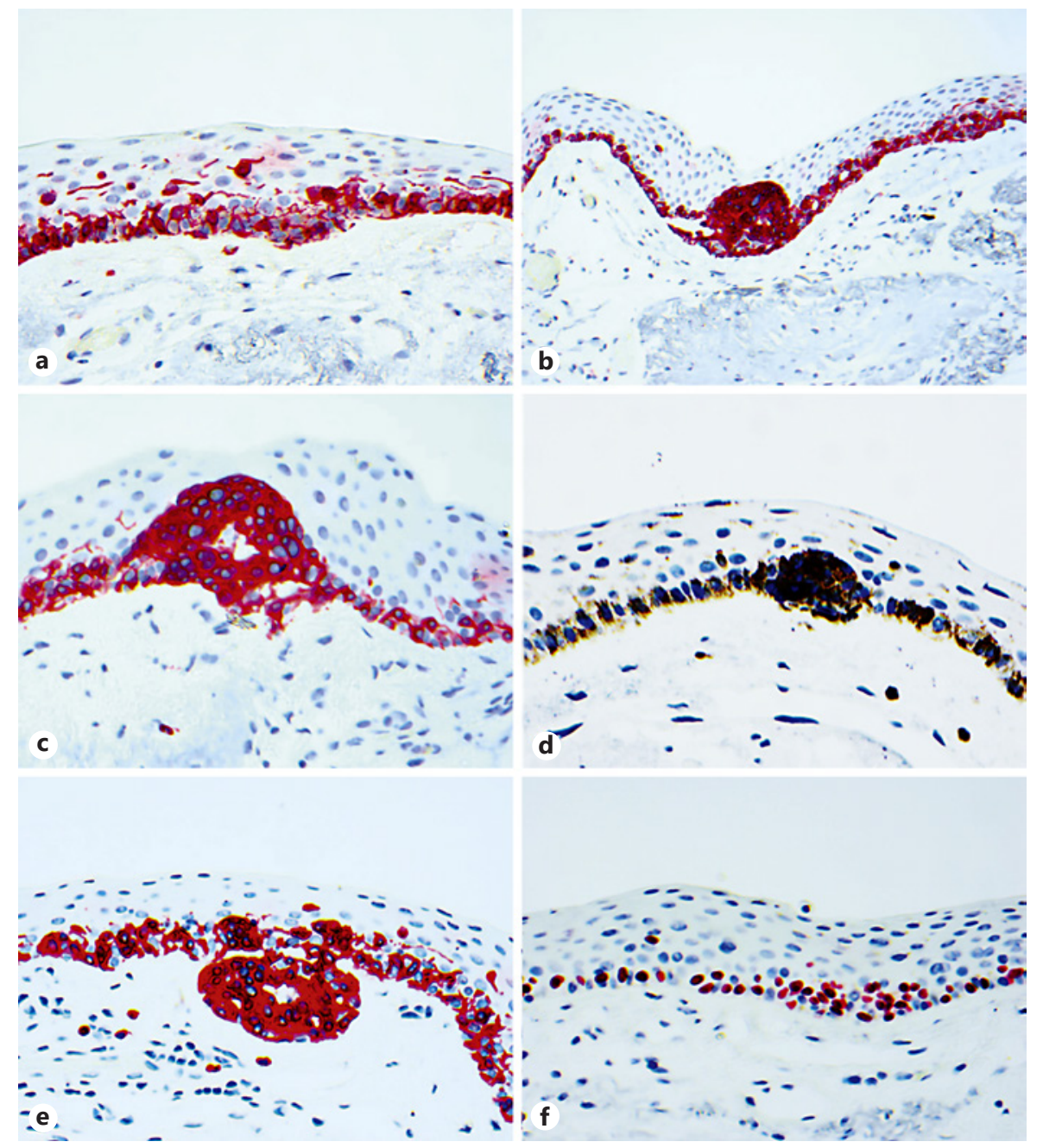

Ocul Oncol Pathol 2020;6:251-258 
Fig. 3. Immunostaining of junctional nests. a SOX-10 stains the nuclei of the nevocytes in a junctional nest and those of the surrounding dendritic melanocytes. The empty spaces in the nest are due to the lack of cytoplasmic staining. b Another positively staining junctional nest stained with SOX10. c SOX-10-positive cells within a junctional nest (arrow) are dropping off into the immediately substantia propria. A separate, deeper nevoid nest (crossed arrow) is unconnected to the epithelium and signifies a focus of early compound nevus formation. d Ki-67 discloses a low proliferation index (red-stained nuclei) among the basal dendritic melanocytes and keratinocytes. e Totally negative Ki-67 staining (absence of red nuclei) is seen in an intraepithelial junction nest. $\mathbf{f}$ A subepithelial nevoid nest is also Ki-67 negative (arrows). a-f Immunoperoxidase, red chromogen. All $\times 40$.

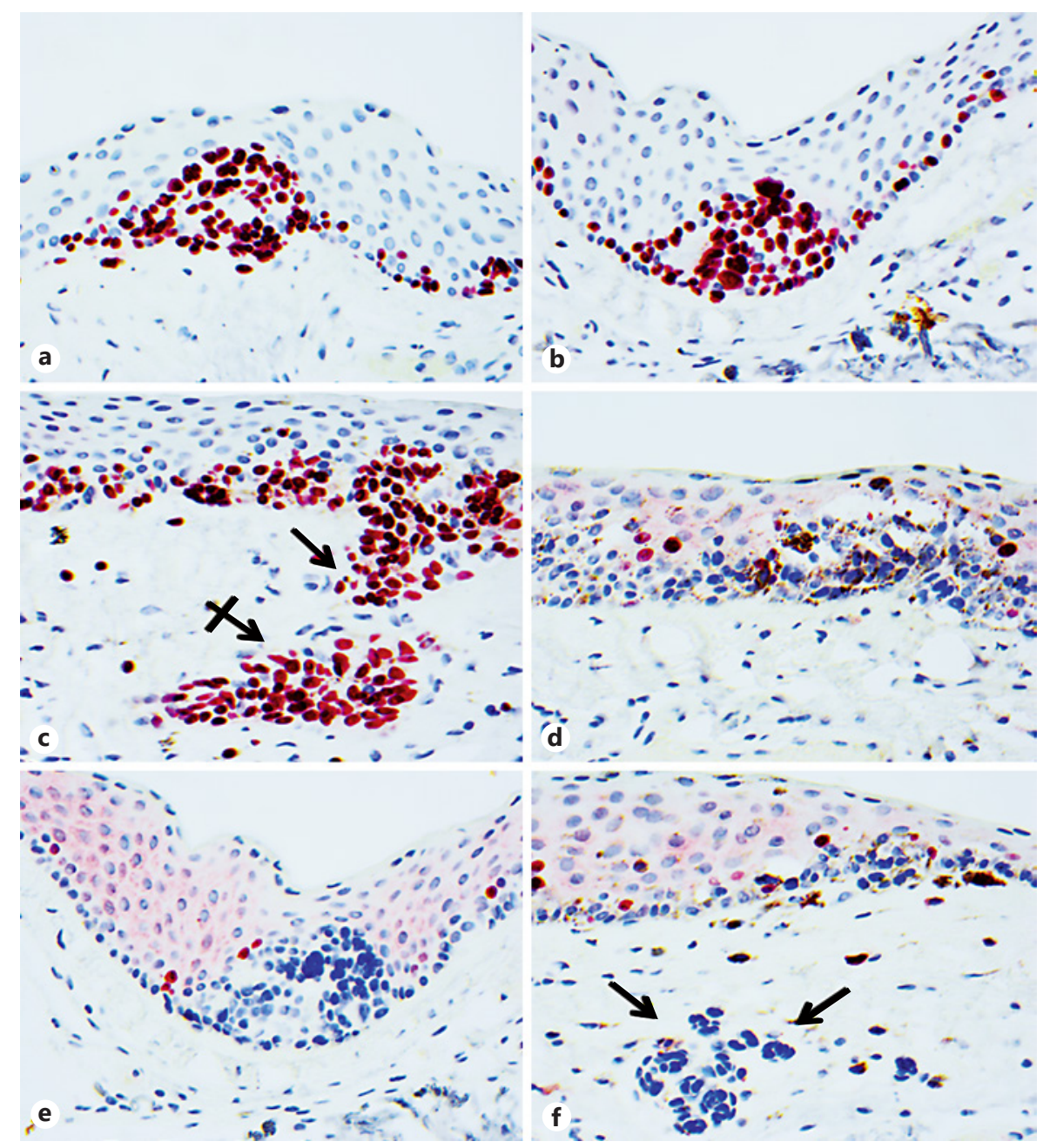

An interesting feature was the formation of junctional nests consisting of rounded collections of nevocytes between the epithelium and the substantia propria (Fig. 1d). These were sharply outlined by MART- 1 and HMB- 45 immunostaining (Fig. 2b-d) and lacked dendrites. Rare small nevoid nests were observed in the superficial substantia propria immediately beneath the epithelium (Fig. 2e). SOX-10 was positive in the nuclei of the basal dendritic melanocytes (Fig. 2f, 3a, b).

One section stained with SOX-10 disclosed the incipient dropping off into the substantia propria of a nest of intraepithelial nevocytes accompanied by an adjacent completely intrastromal nevoid nest (Fig. 3c).

The Ki-67 proliferation index within the epithelium was low $(<2 \%)$ and did not distinguish between keratinocytes or melanocytes. The Ki-67 index was negative in the intraepithelial (Fig. 3d, e) and subepithelial stromal (Fig. 3f) nevoid nests.

\section{Discussion}

Melanocytes are the progeny of the neural crest [1-6], which is a bilobed structure located dorsolateral to the embryonic neural tube. As the neural crest partially breaks up (surviving postnatally as the paraspinal ganglia), the melanocytes that it generates migrate to become terminally lodged ("seeded") within an epithelial layer (epidermotropism) or in a connective tissue ("mesenchymotropism") - for example, the dermis of the skin or the uvea of the eye. The dermal lodgments lead to the formation of blue nevi and the nevus of Ota, while the ocular destinations produce episcleral and uveal melanocytosis. The pigmented cells in the deeper connective tissues are spindled or stellate (multipolar). They are "continent" (their pigment granules are not transferrable to other cells) and generally nonreplicative (postmitotic), never replenishing their melanin granules $[4,6]$. 
The intraepithelial melanocytes are also normally nonreplicative. They have delicate, elongated cell processes (called dendrites) that function as physiologic syringes injecting melanin granules into the surrounding epithelial cells to protect their DNA from ultraviolet radiation (the dendritic melanocytes and keratinocytes of the epidermis or conjunctival epithelium are called the "epidermal-melanin unit"). There is approximately 1 dendritic melanocyte per 5-20 keratinocytes in the skin. Conjunctival intraepithelial dendritic melanocytes are smaller and less conspicuous than those of the epidermis; the density of the former cells has not yet been determined.

Because dendritic melanocytes normally do not proliferate, sun-tanning is the result of the increased melaninization of melanin granule matrices (so-called benign or racial melanosis) as a result of the activity of the enzyme tyrosinase $[7,8]$. If destroyed, dendritic melanocytes do not repopulate the damaged segment of skin (postinflammatory vitiligo, particularly seen in darkly complexioned individuals).

The uveal melanocytes (mesenchymotropic) are also normally nonreplicative, disappearing after an inflammatory chorioretinal event. They are exceptionally responsible, however, for benign spindle cell uveal melanocytic nevi and for much rarer malignant spindle and/ or epithelioid cell melanomas.

Intraepithelial dendritic melanocytes have been considered by many authorities to be a cell population separate from nondendritic nevocytic melanocytes (nevocytes) $[4,5,7-9]$. The latter are believed to migrate separately from dendritic melanocytes and are often held to be the sole precursor of nevocytic nevi (also called common acquired nevi or "moles"). There is a problem, however, with this terminology. For instance, nevi of all the constituents of the skin have been characterized [10]; epidermal, adnexal gland (eccrine and apocrine), adipose tissue, elastic fiber, and vascular nevi (nevus flammeus) have been described. A nevus is a congenital birthmark or skin color blemish. Therefore, we prefer the more accurate and precise term "nevomelanocytic nevus" over the less specific term "nevocytic nevus," since a nevocyte could be a fat cell, a glandular cell, or a vascular endothelial cell.

Ultrastructural findings have clearly established that there are significant morphologic differences between dendritic melanocytes and nevomelanocytic nevocytes [11]. They share features such as the absence of desmosomes between them and the keratinocytes. Nevocytes display a rounded shape with blunt, short villi which are incapable of melanin granule transfer to surrounding keratinocytes. Their melanin granules are smaller due to defective matrices, and they display sporadic granular melanosomal clumping. Dendritic melanocytes have single, dispersed, well-formed melanosomes that become variably melanized on the melanosomal matrices.

There have been several theoretical constructs dealing with the origin of congenital melanocytic nevi and common acquired nevi $[4,6-8]$. Masson believed that the dermal component of both congenital and acquired nevi had a peripheral nerve origin because of the Schwannian or neuroid appearance of spindled cells in the deeper zone of some lesions. Ultrastructural $[8,11]$ and histochemical investigations have furthermore explored the theory of a Schwannian origin, establishing instead that the deep dermal component is composed of modified nevocytes. Other works have postulated activation of persistent local tissue melanoblastic stem cells as an origin for nevi $[6,8]$. A third school of thought is attracted to the possibility of a mutation-engendered conversion of the dendritic melanocyte (perhaps related to ultraviolet radiation) into a nevomelanocyte $[6,8]$. The conspicuous elastotic material seen in the substantia propria of the present lesion lends support for this supposition. A final line of argument that has been pursued is whether nevi are hamartomas. This theory does not comport with the observations of their evolution and involution and that they are mostly acquired rather than congenital. Hamartomas are by definition congenital and by empiric observation stationary throughout life, enlarging only in proportion to the individual's overall progressive growth.

Benign nevocytes have infrequently been discovered in peripheral lymph nodes [12], even reaching the thymus gland [13]. This benign "metastatic phenomenon" further reinforces the proliferative and dyscohesive properties of the altered state of nevomelanocytes, which differs from the stationary behavior of intraepithelial dendritic melanocytes. An early study regarding the clonality of nevocytes initially yielded evidence for polyclonality [14]. Later findings have uniformly led to a consensus that nevomelanocytic nevi are monoclonal $[15,16]$. Evidence of the roles for the BRAF and NRAS gene pathways being partially accountable for the development of nevi and melanomas has also been uncovered $[17,18]$.

The preceding discussion pertains only to postnatal acquired nevi and not to congenital nevi-so-called kissing nevi of the eyelids, bathing trunk nevi, or giant pig- 
mented, hairy nevi of the scalp/face, trunk, or limbs [19]. In these lesions, a bifurcation of cell types can be appreciated, with the nevocytes more extensively involving the dermal collagen, adnexal structures, and subdermal segments of fibroadipose tissue. Such findings could logically be invoked as an example of a truly separate nevocytic population, in contradistinction to an origin from dendritic melanocytes. Genetic studies are underway to establish molecular differences between congenital and acquired nevi [18].

There have been rare cases of common nevi developing de novo in adults, but most garden variety nevi have been posited to be due to the proliferation of nevocytic melanocytes that are present from birth and become activated in the first two decades of life. Common nevi $[7-9,20]$ evolve from intraepithelial junctional nevocytic nests of closely clustered cells or "theques." These nevocytic clusters rest on the epithelial basement membrane at the "junction" of the epithelium with the underlying connective tissue (junctional nests). The nevomelanocytes are extruded from the epidermis to drop off ("abtropfung" phenomenon) into the underlying connective tissue (the papillary dermis in the skin, the substantia propria in the mucosae). This accounts for the formation of compound nevi. The intraepithelial junctional nests ultimately burn out, leaving behind a dermal or subepithelial nevoid cellular mass that may involute with aging through progressive fibrotic replacement [21].

The present lesion challenges the hard-and-fast distinction between dendritic melanocytes and nevomelanocytic (nevocytic) melanocytes. The basic lesion was predominantly constituted by a proliferation of intraepithelial basal dendritic melanocytes, thereby implying at least benign hyperplasia, since - as already mentioned - normal intraepithelial dendritic melanocytes do not make direct contact with each other. Whether this process in our case was premalignant or a form of hyperplasia cannot be answered at this time due to the absence of a reliable distinguishing biomarker (only when there is frank cytologic atypia can a bona fide neoplasm be safely deduced). The dendritic melanocytic proliferation in the current lesion was prominent in the center and trailed off at the edges of the excision. In the most pronounced proliferative zones, occasional dendritic melanocytes percolated to the mid-level of the epithelium or tended to crowd together on or above the basement membrane at the epithelial-mesenchymal junction, where they created tangles of their dendritic processes.
The interesting finding was the population of rounded, intraepithelial, relatively nonpigmented cells that were organized into well-defined, cohesive nests (theques) with surrounding haloes or cavities. This retraction artifact was due to the absence of melanocytic desmosomes and tonofilaments. In one fortuitous section, a collection of intraepithelial nevomelanocytes was continuous through a break in the basement membrane with cells in the immediate subepithelial zone of the substantia propria (Fig. 2e), highlighting the latter's extrusion from within the epithelium into the underlying connective tissue (signifying the earliest phase of compound nevus formation) [7-9].

Our interpretation of the current lesion is that it represents an example of an intraepithelial dendritic melanocytic proliferation [22] without atypia. For several decades, "primary acquired melanosis" was the preferred term [22-29]. Another designation for this lesion that has recently been introduced is "conjunctival intraepithelial neoplasia" [30]. The problem with this last term is that it does not allow for the state of melanocytic hyperplasia $[31,32]$. In the absence of frank cytologic atypia, it must be understood that there is currently no immunohistochemical biomarker that facilitates the separation of intraepithelial hyperplasia from early atypical proliferation. Therefore, all increased numbers of intraepithelial melanocytes in the conjunctival intraepithelial neoplasia scheme are often automatically - but probably erroneously - lumped together as a clonal and potentially premalignant neoplasm. Lesions exhibiting severe cytologic atypia have also been referred to as "melanomas in situ" $[33,34]$ in the preinvasive state.

Within the current lesion, there developed the earliest stage of a conventional nevomelanocytic nevus with incipient compound nevus formation. Both nevi and melanomas have been correlated with ultraviolet light (sun) exposure, consistent with our patient's pronounced lesional elastotic material in the substantia propria without squamous epithelial atypia. It is most unusual, however, for a nevus to first appear in late adulthood (the current patient was 52 years old at the time of the excision of his lesion). In contrast, intraepithelial dendritic melanocytic proliferation/primary acquired melanosis/conjunctival intraepithelial neoplasia characteristically arises in mid-life or later (average age around 62 years) [34], with which our patient's age is consistent. The current lesion is anomalous because the usual scenario and sequence is that around $30 \%$ of cutaneous and conjunctival melanomas arise first from a 
preexistent nevus after development of intraepithelial (pagetoid spread) atypia [17]; the opposite situation occurred in the current lesion. In the usual progression from a nevus to a melanoma there is persistent junctional activity that transforms by sequential clonal expansions into a radial intraepithelial growth phase (pagetoid spread) [26]. A final mutation results in invasion of the underlying connective tissue (either dermis or substantia propria) to create a nodule of melanoma. The dermal component of nevi is generally not responsible for melanomas, since its cells are postmitotic.

Our conclusion is that proliferating dendritic melanocytes can spawn, at least in some situations and conditions, a secondary nevomelanocytic population, which in turn can evolve according to the classic model into a conventional compound nevus. This case provides some proof of an alternative origin of nevocytic melanocytes as opposed to an embryologically separate population of cells ab initio. It may also help to explain the remarkable event of the late onset of a common acquired conjunctival nevus in mature adults from dendritic melanocytes.

\section{Statement of Ethics}

This study complies with the tenets of the Declaration of Helsinki and Health Insurance Portability and Accountability Act regulations. All surgical informed consent forms signed by the patient at our institution contain an approval for the clinical material to be used in any future publications.

\section{Disclosure Statement}

The authors have no financial or proprietary interests in the materials mentioned herein. No conflicting relations exist for any author.

\section{Funding Sources}

Department of Ophthalmology Research Fund, Massachusetts Eye and Ear. This research did not receive any specific grant from funding agencies in the public, commercial or non-profit sectors.

\section{Author Contributions}

Frederick A. Jakobiec: main author; responsible for supervision, conceptual guidance, and writing of the manuscript. Paula Cortes Barrantes: responsible for data summary, preparation, library search, references, and illustrations. Mary E. Aronow: provided the case report and patient follow-up.

\section{References}

1 Jakobieg FA, Tannenbaum M. Embryological perspectives on the fine structure of orbital tumors. Int Ophthalmol Clin. 1975;15(1):85-110.

2 Vandamme N, Berx G. From neural crest cells to melanocytes: cellular plasticity during development and beyond. Cell Mol Life Sci. 2019 May;76(10):1919-34.

3 Martik ML, Bronner ME. Regulatory logic underlying diversification of the neural crest. Trends Genet. Trends Genet. 2017 Oct; 33(10):715-27.

4 Quevedo WC Jr, Fleischmann RD. Developmental biology of mammalian melanocytes. J Invest Dermatol. 1980 Jul;75(1):116-20.

5 Weston JA. Lineage commitment and fate of neural crest-derived neurogenic cells. Adv Pharmacol. 1998;42:887-91.

6 Robinson WA, Lemon M, Elefanty A, Harrison-Smith M, Markham N, Norris D. Human acquired naevi are clonal. Melanoma Res. 1998 Dec;8(6):499-503.

7 Weedon D. Lentigines, nevi and melanomas. In: Weedon D, editor. Weedon's skin pathology. 3rd ed. China: Churchill-Livingstone; 2010. pp. 713-7.

8 Elder DE, Elenitsas R, Murphy G, Xu X. Benign pigmented lesions and malignant melanoma. In: Elder DE, Elenitsas R, Rosenbach M, editors. Lever's histopathology of the skin. 11th ed. Philadelphia: Wolters Kluwer; 2015. pp. 872-3.

9 Jakobiec FA, Rootman J, Jones IS. Secondary and metastatic tumors of the orbit. In: Jakobiec FA, Jones IS, editors. Diseases of the orbit. 1st ed. Hagerstown (MD): Harper \& Row; 1979. pp. 517-22.

10 Callahan AB, Jakobiec FA, Zakka FR, Fay A. Isolated unilateral linear epidermal nevus of the upper eyelid. Ophthal Plast Reconstr Surg. 2012 Nov-Dec;28(6):e135-8.

11 Jakobiec FA. The ultrastructure of conjunctival melanocytic tumors. Trans Am Ophthalmol Soc. 1984;82:599-752.

12 Biddle DA, Evans HL, Kemp BL, El-Naggar AK, Harvell JD, White WL, et al. Intraparenchymal nevus cell aggregates in lymph nodes: a possible diagnostic pitfall with malignant melanoma and carcinoma. Am J Surg Pathol. 2003 May;27(5):673-81.

13 Parker JR, Ro JY, Ordóñez NG. Benign nevus cell aggregates in the thymus: a case report. Mod Pathol. 1999 Mar;12(3):329-32.

14 Harada M, Suzuki M, Ikeda T, Kaneko T, Harada S, Fukayama M. Clonality in nevocellular nevus and melanoma: an expression-based clonality analysis at the $\mathrm{X}$-linked genes by polymerase chain reaction. J Invest Dermatol. 1997 Nov;109(5):656-60.
15 Hui P, Perkins A, Glusac E. Assessment of clonality in melanocytic nevi. J Cutan Pathol. 2001 Mar;28(3):140-4

16 Indsto JO, Cachia AR, Kefford RF, Mann GJ $\mathrm{X}$ inactivation, DNA deletion, and microsatellite instability in common acquired melanocytic nevi. Clin Cancer Res. 2001 Dec;7(12): 4054-9.

17 Damsky WE, Bosenberg M. Melanocytic nevi and melanoma: unraveling a complex relationship. Oncogene. 2017 Oct;36(42):5771-92.

18 Roh MR, Eliades P, Gupta S, Tsao H. Genetics of melanocytic nevi. Pigment Cell Melanoma Res. 2015 Nov;28(6):661-72.

19 Elder DE, Murphy GE. Melanocytic tumors of the skin. In: Silverberg ST, Gardner WA, Sobin LH, editors. Armed Forces Institute of Pathology, Atlas of Tumor Pathology 12. 4th ed. Washington (D.C.): American Registry of Pathology; 2010. pp. 135-62.

20 Crowson AN, Magro CM, Mihm MC Jr. Benign acquired nevi. In: Crowson AN, Magro CM, Mihm MC Jr, editors. The melanocytic proliferations: a comprehensive textbook of pigmented lesions. 2nd ed. New Jersey: Wiley-Blackwell; 2014. pp. 65-84.

21 Maize JC, Foster G. Age-related changes in melanocytic naevi. Clin Exp Dermatol. 1979 Mar;4(1):49-58. 
22 Jakobiec FA. Conjunctival primary acquired melanosis: is it time for a new terminology? Am J Ophthalmol. 2016 Feb;162:3-19.e1.

23 Jakobiec FA, Folberg R, Iwamoto T. Clinicopathologic characteristics of premalignant and malignant melanocytic lesions of the conjunctiva. Ophthalmology. 1989 Feb;96(2): 147-66.

24 Jakobiec FA. Naming the precursors of conjunctival melanoma. Am J Ophthalmol. 2016 Apr;164:152-3.

25 Liesegang TJ, Campbell RJ. Mayo Clinic experience with conjunctival melanomas. Arch Ophthalmol. 1980 Aug;98(8):1385-9.

26 Jakobiec FA. Conjunctival melanoma. Arch Ophthalmol. 1980 Aug;98(8):1378-84.
27 Folberg R, McLean IW, Zimmerman LE. Primary acquired melanosis of the conjunctiva. Hum Pathol. 1985 Feb;16(2):129-35.

28 Folberg R, McLean IW. Primary acquired melanosis and melanoma of the conjunctiva: terminology, classification, and biologic behavior. Hum Pathol. 1986 Jul;17(7):652-4.

29 Folberg R. Naming the precursors of conjunctival melanoma. Am J Ophthalmol. 2016 Feb; 162:1-2.

30 Damato B, Coupland SE. Conjunctival melanoma and melanosis: a reappraisal of terminology, classification and staging. Clin Exp Ophthalmol. 2008 Nov;36(8):786-95.
31 Coupland SE, Damato BE. Conjunctival primary acquired melanosis: is it time for a new terminology? Am J Ophthalmol. 2016 May; 165:203-4.

32 Grossniklaus HE, Eberhart CG, Kivela TT. Conjunctival melanocytic intraepithelial neoplasia. In: Grossniklaus HE, Eberhart CG, Kivela TT, editors. WHO classification of tumors of the eye. 4th ed. Lyon: International Agency for Research on Cancer (IARC); 2018. pp. 31-4.

33 Ackerman AB, Sood R, Koenig M. Primary acquired melanosis of the conjunctiva is melanoma in situ. Mod Pathol. 1991 Mar;4(2): 253-63.

34 Folberg R, Jakobiec FA, McLean IW, Zimmerman LE. Is primary acquired melanosis of the conjunctiva equivalent to melanoma in situ? Mod Pathol. 1992 Jan;5(1):2-5; discussion 6-8. 